\title{
Assessment of Renal Length and Serum Creatinine Values in Chronic Kidney Disease - A Hospital Based Study
}

\author{
S. K. Y. I. Kodikara ${ }^{1}$, D. T. K. Gamage 2 , L. Y. D. Dulmini ${ }^{3}$, B. G. Nanayakkara ${ }^{1}$, \\ I. Ilayperuma ${ }^{1}$
}

\begin{abstract}
Introduction: Chronic kidney disease (CKD) is a global \& local health problem. Serum creatinine (Scr) is an indicator of renal function. Ultrasound is a freely available safe, reliable imaging modality, commonly used to assess kidneys in CKD. Association of renal length and Scr in CKD is evaluated in this study, as such data is lacking for Sri Lankan population. Methodology: A descriptive cross sectional observational study was conducted at the Radiology Unit of the Base Hospital Tissamaharama from March to August 2017 (as a preliminary study of a large ongoing study), using 41 patients with normal Scr (control group) and 74 patients diagnosed with CKD. The Scr values done within the last three months were evaluated. Absolute renal length was measured ultrasonically by an experienced Radiologist, who was blind to the patient's serum creatinine value, with Mind-ray ultra sound scanner.
\end{abstract}

Results: Study population $(n=115)$ consists of control group $(n=41)$ and CKD patients $(n=74)$ where $40 \%$ were females and $60 \%$ of were males. Mean age was 58.3 years \& mean renal length in control group was $9.6 \mathrm{~cm}$ and CKD group was $9.0 \mathrm{~cm}$. A significant difference observed in renal length in control and CKD groups $(<0.001) \&$ a weak negative correlation between renal length and Scr value in CKD group ( $\mathrm{r}=0.328)$.

Conclusion: Absolute renal length in CKD was significantly lower compared to the control group. Weak negative correlation was established between absolute renal length and the Scr value, thus with the renal function. Further studies are needed to evaluate the relationship between relative renal length and serum creatinine values and to assess above parameters according to etiological categories of CKD.

Key words: chronic renal failure, serum creatinine, ultrasound scan, renal length.

\section{INTRODUCTION}

Chronic kidney disease (CKD) is defined as reduced renal function for three or more months (1). CKD is a global health problem with a prevalence of $13.4 \%$ $(11 \cdot 7-15 \cdot 1 \%)$ (2). It is becoming a major health problem in Sri Lanka over last two decades and a significant burden to the health sector.

Ultrasound (USS) is a safe imaging modality freely available in developing countries like Sri Lanka, which does not involve with ionizing radiation. Sonographical measurement of renal length is an easy, reproducible and non invasive method. According to the available literature, no significant inter and intra observer renal measurement variability with USS, thus is a reliable imaging modality for assessment of renal length (3). Other available imaging

\footnotetext{
${ }^{1}$ Department of Anatomy, Faculty of Medicine, University of Ruhuna, ${ }^{2}$ Consultant Radiologist, Base hospital Tissamaharama,

${ }^{3}$ Medical officer, Base hospital Tissamaharama.
} 
KODIKARA, S. K. Y. I., GAMAGE, D. T. K., DULMINI, L.Y. D., NANAYAKKARA, B. G., ILAYPERUMA, I., Assessment of renal length and serum creatinine levels in chronic kidney disease - A hospital based study. Sri Lanka Anatomy Journal (SLAJ), 1(2): 22-27, 2017.

modalities like computer tomography, provide promising answer for renal measurements, with the cost of ionizing radiation exposure. For said reasons, renal USS is of value in the assessment of renal size in CKD patients $(3,4,5,6)$. Population specific analysis of renal size is important, as renal size varies with ethnicity (7).

Serum creatinine (Scr) value is an indicator of renal function and cut off values are established to evaluate renal function and to diagnose renal impairment (8).

The present study was aimed at evaluating the association between absolute renal length and serum creatinine values in CKD and to compare with that of healthy population.

\section{MATERIALS AND METHOD}

Present descriptive cross sectional observational study was approved by the ethical review committee of the Faculty of Medicine, University of Ruhuna and was conducted at the Radiology Unit of the Base Hospital Tissamaharamaya from March to August 2017 as a preliminary study of a large ongoing study. Present study included 41 patients with normal serum creatinine as control group and 74 patients diagnosed with CKD. Study subjects were above 18 years of age. The serum creatinine reports that were done within the last three months, at the day lab of the Base Hospital Tissamaharamaya were included in this study. Following patients were excluded from the study; history of previous renal surgeries, acute renal insufficiency or on renal replacement therapy (haemodialysis, peritoneal dialysis, renal transplantation), fatty liver or any other liver disease and unwilling patients. All USS were performed by an experienced Radiologist, who was blind to patients serum creatinine value, with Mind-ray ultra sound scanner, using standard grey scale imaging with $3.5 \mathrm{MHz}$ curved array transducer. Grey scale amplification and the time gain compensation curve were adjusted to acquire the best quality images. Single focus point was adjusted at the level of the kidney, while routinely using tissue harmonic imaging. All patients were well hydrated and are with full bladder at the time of imaging. Maximum pole to pole length (Figure 1) of each kidney was measured and recorded as the renal length (absolute).

Descriptive analysis of data was done with mean, standard deviation, minimum and maximum values. Student's t-test was used to evaluate the differences between two groups. Correlation between the variables was assessed with Pearson correlation coefficient.

\section{RESULTS}

Study population $(n=115)$ consisted of a control group $(n=41)$ and diagnosed CKD patients $(n=74)$. Forty six $(40 \%)$ of them were females and 69 (60\%) were males. Average age was 58.3 years (range 20 - 89 years). Average renal length in the control and CKD groups are shown in Table 1. The results of Student t-test comparing the renal lengths of control and CKD groups are presented in Table 2. Serum creatinine values of respective groups are shown in Table 3. Figure 2 demonstrates the relationship between the renal length and serum creatinine values. Pearson correlation co-efficient comparing serum creatinine values with renal length in CKD group shows weak negative correlation, $r$ value was -0.328 . 


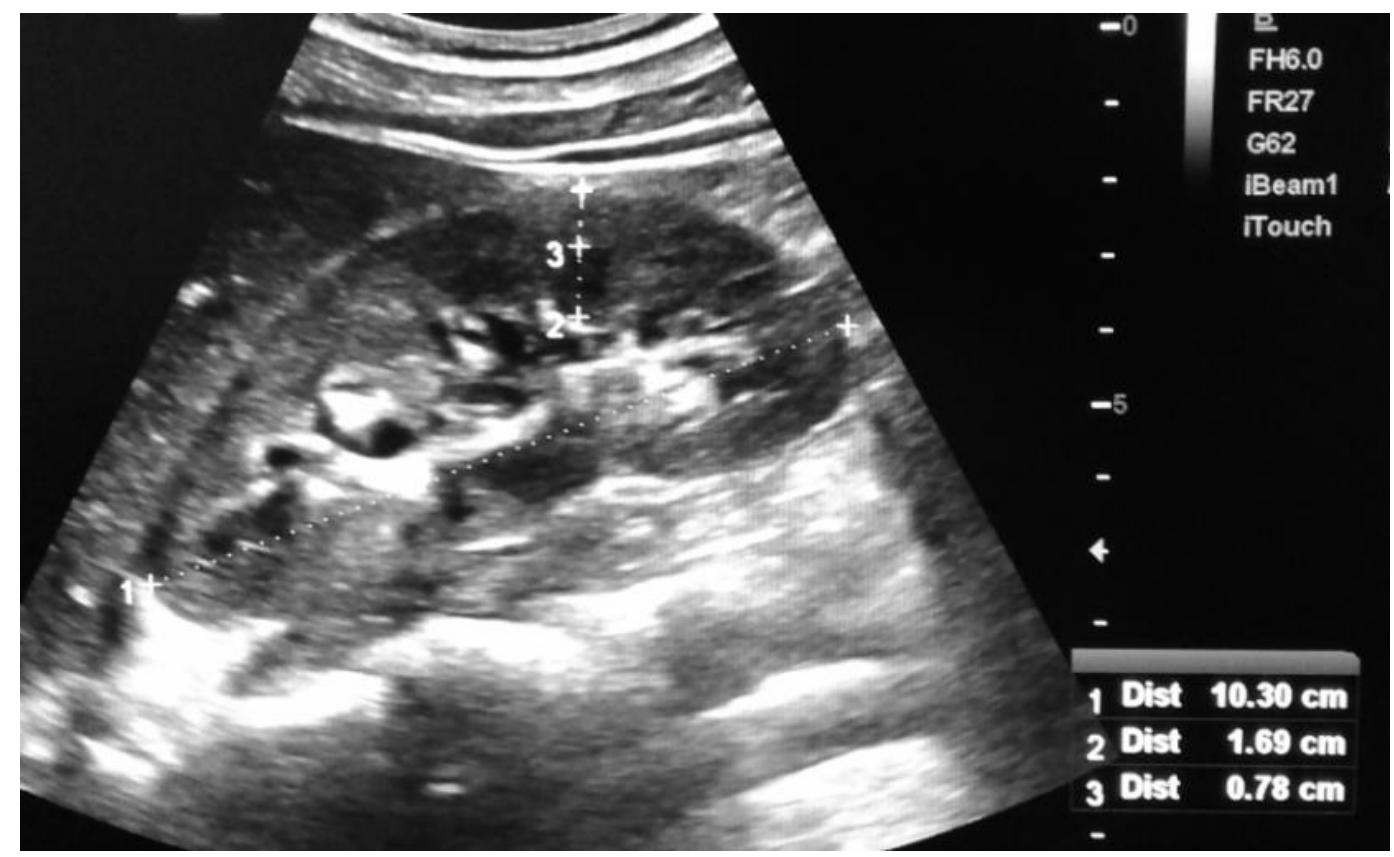

Figure 1: Renal length measurement using Ultrasound

1-Renal length, 2-Medullary thickness, 3-Cortical thickness

Table 1. Average renal length in the control and CKD groups

\begin{tabular}{ccc}
\hline Renal length $(\mathbf{c m})$ & Control group & CKD \\
\hline Right renal length. mean \pm SD & $9.98 \pm 0.87$ & $8.97 \pm 1.3$ \\
Right renal length: range & $6.5-11.1$ & $6.8-11.4$ \\
Left renal length: mean \pm SD & $9.94 \pm 0.75$ & $9.0 \pm 1.3$ \\
Left renal length: range & $8-11.2$ & $8.2-11.2$ \\
\hline
\end{tabular}

Table 2. Student t-test results comparing renal lengths of control and CKD groups

\begin{tabular}{lccc}
\hline & T value & P value & Sig/ N.sig \\
\hline RK, control Vs CKD & 5.29 & $<0.00001$ & Significant \\
LK, control Vs CKD & 4.89 & $<0.00001$ & Significant \\
\hline
\end{tabular}

$\mathrm{P}$ value $<0.01$ is considered as significant. RK- right kidney; LK - left kidney 
KODIKARA, S. K. Y. I., GAMAGE, D. T. K., DULMINI, L.Y. D., NANAYAKKARA, B. G., ILAYPERUMA, I., Assessment of renal length and serum creatinine levels in chronic kidney disease - A hospital based study. Sri Lanka Anatomy Journal (SLAJ), 1(2): 22-27, 2017.

Table 3. Serum creatinine values of control \& CKD groups

\begin{tabular}{ccc}
\hline Serum creatinine $(\mathrm{mg} / \mathrm{dL})$ & Control group & CKD \\
\hline Mean serum creatinine $\pm \mathrm{SD}$ & $0.83 \pm 0.15$ & $1.89 \pm 0.55$ \\
Serum creatinine range & $1.1-1.6$ & $4.0-1.4$ \\
\hline
\end{tabular}

\section{DISCUSSION}

Natural history of CKD demonstrates a progressive decline in renal size with progressive loss of renal function $(6,5)$. Present study analyzed the relationship between the sonograhically measured absolute renal length and serum creatinine value. Serum creatinine, an indicator of renal function, used to compare renal function and renal length in CKD and control groups. The results of the present study established a statistically significant deference in renal length between the two study groups, CKD and control groups. The renal length of the CKD group was

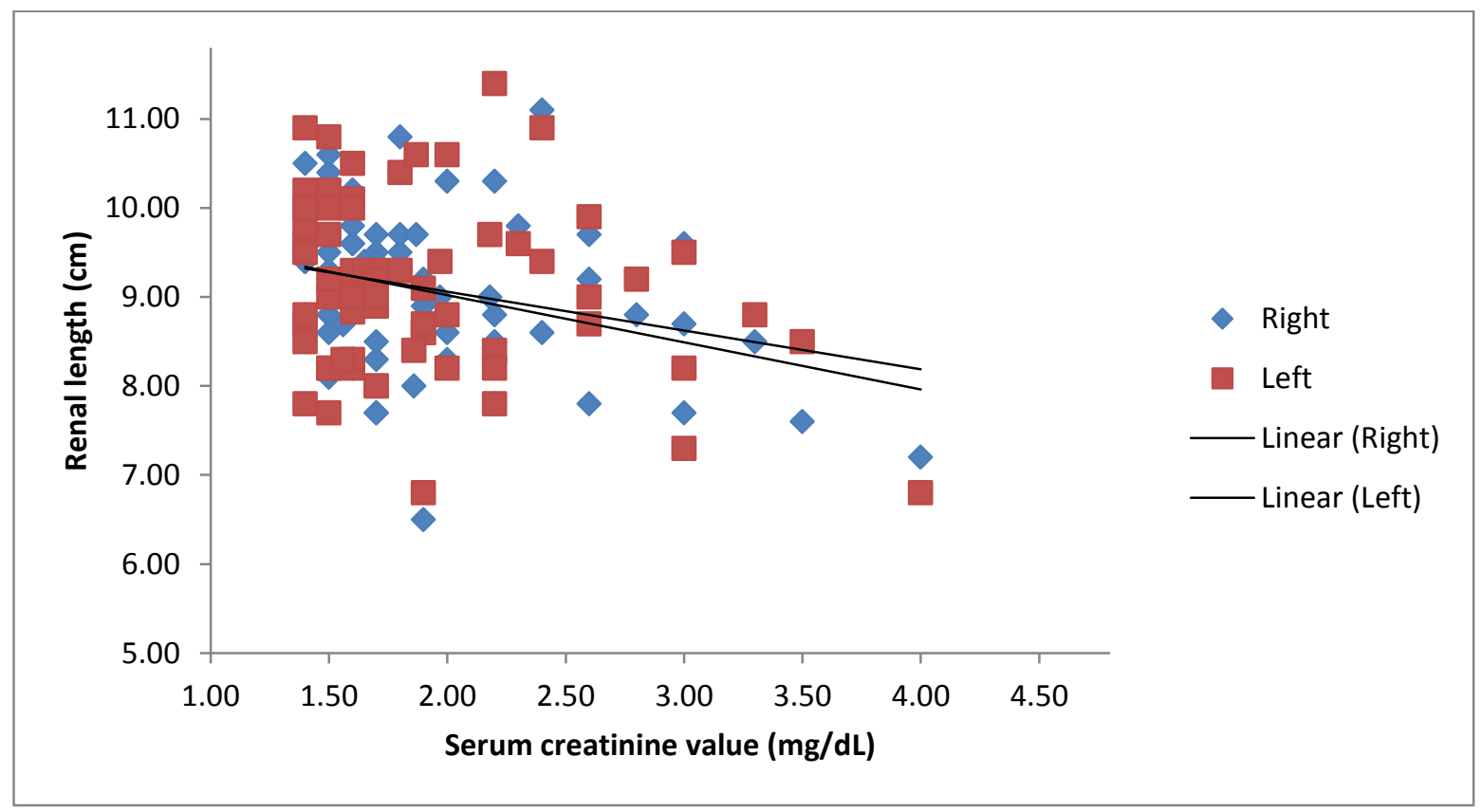

Figure 2: Serum creatinine value Vs renal length

significantly lower than that of the control group. This finding is in par with the results of previous similar studies (4). There was no significant difference in the renal length between right \& left kidneys either within the control or within the CKD groups. A weak negative correlation (r- 0.328) was established between the renal lengths and Scr values.

Jovanovic et al. reported a strong negative correlation (-0.69) of the same parameters. They studied 49 CKD patients and 46 healthy adults as a control group (9). As in the present study, they have also not 
KODIKARA, S. K. Y. I., GAMAGE, D. T. K., DULMINI, L.Y. D., NANAYAKKARA, B. G., ILAYPERUMA, I., Assessment of renal length and serum creatinine levels in chronic kidney disease - A hospital based study. Sri Lanka Anatomy Journal (SLAJ), 1(2): 22-27, 2017.

catagcategorized CKD patients according to the etiology of renal disease, therefore a similar methodology. Some authors have described an etiology related variation in renal length in CKD patients (4). Kokoris et al. have studied 126 patients with chronic kidney diseases with different etiologies, including Balkan endemic nephropathy, diabetic nephropathy, autosomal dominant polycystic kidney disease, primary glomerular disease and compared renal length with a control group. They have established a significant difference in renal lengths with different etiologies. Diabetic nephropathy (DMN) is recognized as a common cause of CKD in Sri Lanka (10), therefore it is assumed that our study sample must have a significant number of DMN. In DMN, the renal size initially increases (4). Therefore, the established weak negative correlation of renal length and Scr in the present study could be partially due to this etiological variation. However, considering the fact that Jovanovic et al. have reported strong negative correlation in above parameters with similar methodology a population specific variability of renal length might play a role in the findings of the current study.

Absolute renal length was measured in the present study as maximum pole to pole to length of the kidneys. Previous studies have established a positive relationship of renal size with the height of the patient $(4,9,11,12)$. In their studies, the relative renal length was calculated by dividing the absolute renal length (in millimeters) by the body height (in centimeters). They concluded that relative renal length as a better indicator of renal length compared to absolute renal length. Therefore, it will be of value to assess the relative renal length in order to investigate the association of renal length and renal function.

\section{CONCLUSION}

Findings of the current study established a significant reduction in absolute renal length in CKD patients when compared to the control group.

Weak negative correlation was elicited between the absolute renal length and the serum creatinine value in CKD, therefore with the renal function.

Further studies are recommended to evaluate the relationship between the relative renal length and serum creatinine values and to assess above parameters according to etiological categories of CKD.

\section{REFFERENCES}

1. American Association of Family Physician. Clinical Practice Guidelines. 2004: 70:869-86.

2. Albert J, Coulthard A, Lee REJ et. al. How reliable are ultrasound measurements of renal length in adults. The British Journal of Radiology 1995: 1087-1089.

3. El-Reshaid W, Abdul-FattahH. Sonographic Assassment of Renal Size in Healthy Adults. Medical Principles and Practice, 2014: 8:432-436.

4. Kokoris JK., Vlajkovic S, Absolute and relative kidney length in chronic renal disease, Acta Medica Medianae, 2015:1723.

5. O'Neill, Charles W, Renal Relevant Radiology: Use of Ultrasound in Kidney Disease and Nephrology Procedures,: American Society of Nephrology, 2014.

6. Gareeballah A, Gameraddin M, Mustafa $\mathrm{H}$, Alshabi S, Sonographic findings in renal parenchymal diseases at Sudanese. Open Journal of Radiology, 2015: 7:243249.

7. Arooj A, Lam J, Wui YJ, Supriyanto E. Comparison of Renal Size among Different Ethnicities. International Journal for biology and biomedical engineering, 2011:18: 221-229. 
8. Couchoud C, Pozet N, Labeeuw M, Pouteil-Noble C: Screening early renal failure: Cut-off values for serum creatinine as an indicator of renal impairment.Kidney Int: 1999, $1878-1884$.

9. Jovanovic D, Gasic B, Pavlovic S, Naumovic R. Correlation of kidney size with kidney function andanthropometric parameters in healthy subjects and patients with chronic kidney disease, Renal failure, 2013: 896-900.

10. Gooneratne IK., Ranaweera AKP, Liyanarachchi NP, Gunawardane N, Lanerolle RD. Epidemiology of chronic kidney disease in a Sri Lankan population. International Journal of Diabetes in Developing Countries, 2008:12: 60-64.
11. Miletić D, Fuckar Z, Sustić A, Mozetic V, Stimac D, Zauhar G. Sonographic measurement of absolute and relative renal lenght in adults. $J$ Clin Ultrasound, 1998:185-189.

12. Hekmatnia A, Yaraghi M. Sonographic measurement of absolute and relative renal length in healthy Isfahani adults. $J$ Res Med Sci , 2004:28: 54-57.

\section{CORRESPONDENCE}

Dr. S. K. Y. I. Kodikara

Department of Anatomy, University of Ruhuna.

E-mail-iroshani.kodikara@gmail.com

Received: September 2017

Accepted: September 2017 